Reliability Engineering \& System Safety Volume 91, Issues 10-11, OctoberNovember 2006, Pages 1364-1372 The Fourth International Conference on Sensitivity Analysis of Model Output (SAMO 2004) - SAMO 2004

doi:10.1016/j.ress.2005.11.054

Copyright (c) 2005 Elsevier Ltd All rights reserved.

\title{
Probabilistic inversion for chicken processing lines
}

\section{Roger M. Cooke ${ }^{a, *}$, Maarten Nautab ${ }^{b}$ Arie H. Havelaar ${ }^{b}$ and Ine van der Fels ${ }^{\mathbf{b}}$}

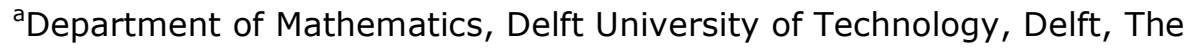
Netherlands

${ }^{\mathrm{b}}$ Microbiological Laboratory for Health Protection RIVM, Bilthoven, The

Netherlands

* Corresponding author. Tel.: +31 15 2782548; fax: +31 152787255 .

\begin{abstract}
We discuss an application of probabilistic inversion techniques to a model of campylobacter transmission in chicken processing lines. Such techniques are indicated when we wish to quantify a model which is new and perhaps unfamiliar to the expert community. In this case there are no measurements for estimating model parameters, and experts are typically unable to give a considered judgment. In such cases, experts are asked to quantify their uncertainty regarding variables which can be predicted by the model. The experts' distributions (after combination) are then pulled back onto the parameter space of the model, a process termed "probabilistic inversion". This study illustrates two such techniques, iterative proportional fitting (IPF) and PARmeter fitting for uncertain models (PARFUM). In addition, we illustrate how expert judgement on predicted observable quantities in combination with probabilistic inversion may be used for model validation and/or model criticism.
\end{abstract}

\section{Introduction}

Campylobacter contamination of chicken meat may be responsible for up to $40 \%$ of the annual 100,000 cases of campylobacter-associated gastroenteritis in The Netherlands, and a similar proportion of an estimated 30 deaths. A recent effort to rank various control options for campylobacter contamination of chicken carcasses has led to the development of a mathematical model of a typical chicken processing line [1] and [2]. This model has been quantified in an expert judgment study involving 12 experts [3]. Key parameters in the model are transfer coefficients from the chickens' skin and intestines to the processing environment, and from the environment back to the chickens' skin. Experimental data on such transfer coefficients is not available, and experts are unable to quantify their uncertainty on the values of these coefficients. Hence, the model must be quantified by asking the experts about other quantities which, under specific circumstances, can be predicted by the model. These quantities typically involve aggregate phenomena with which experts are sufficiently familiar to render a judgment. The experts need not endorse or even know the model. Their uncertainty distributions are combined to form a "decision maker's" distribution, as described in [3]. The problem then arises of "pulling back" the decision maker's distributions onto the parameter space of the model. This is an example of probabilistic inversion and forms the focus of this article. Probabilistic inversion is increasingly used in risk and consequence analysis. Recent applications may be 
Reliability Engineering \& System Safety Volume 91, Issues 10-11, OctoberNovember 2006, Pages 1364-1372 The Fourth International Conference on Sensitivity Analysis of Model Output (SAMO 2004) - SAMO 2004

found in [4], [5], [15] and [16], for a discussion see [6] and [7]. A complete mathematical discussion of the techniques employed here is found in [8].

The 'pull-back' distribution on model parameters may be pushed through the model to re-predict the quantities assessed by the decision maker. This provides an opportunity for model validation and/or criticism. If the re-predicted

distributions agree with the original decision maker's distributions, then the model provides a suitable vehicle for capturing the decision maker's uncertainty. If these distributions do not agree, then the model is not suitable to represent the decision maker's uncertainty. In this case the model must be re-evaluated and possibly revised. For more discussion and examples of this aspect see [5]. The present study illustrates this, and thereby underscores the value of structured expert judgment in model criticism, even when the experts are unfamiliar with the model.

This article begins with a brief description of the mathematical model for chicken processing lines. A non-technical discussion of probabilistic inversion techniques based in iterative algorithms is then given. Finally results with the chicken processing expert data are presented. A final section draws conclusions.

\section{The chicken processing line}

A schematic representation of a typical broiler chicken processing line is given in Fig. 1.

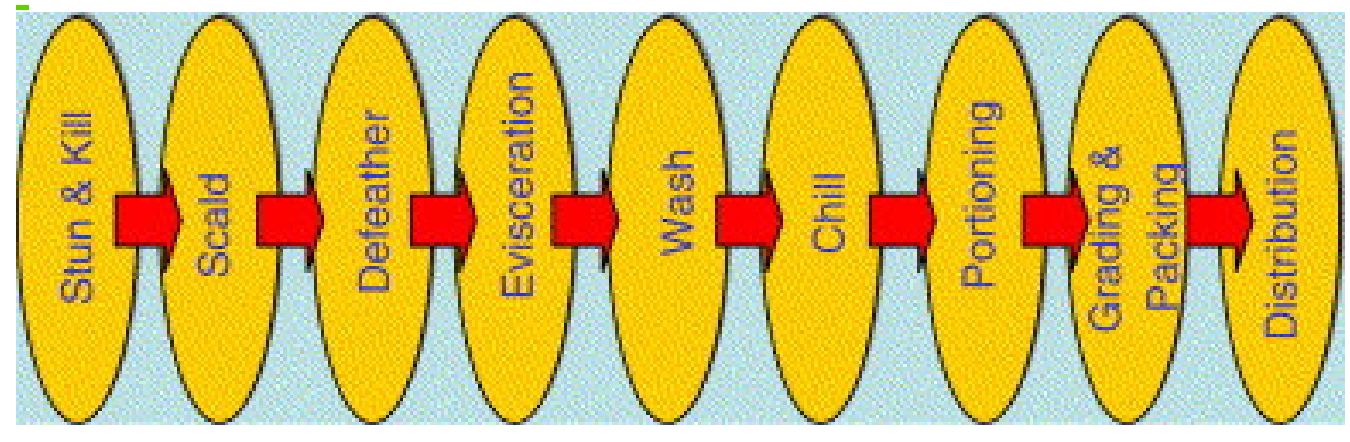

Fig. 1. Broiler chicken processing line.

For campylobacter transmission, the relevant phases are Scalding, defeathering, evisceration, washing, and chilling. Two types of scalding processes are considered, namely low and intermediate temperature, as two types of chilling, namely air and spray chilling. Each phase is modelled as a physical transport process. A typical phase in the processing line is illustrated in Fig. 2. 
Reliability Engineering \& System Safety Volume 91, Issues 10-11, OctoberNovember 2006, Pages 1364-1372 The Fourth International Conference on Sensitivity Analysis of Model Output (SAMO 2004) - SAMO 2004

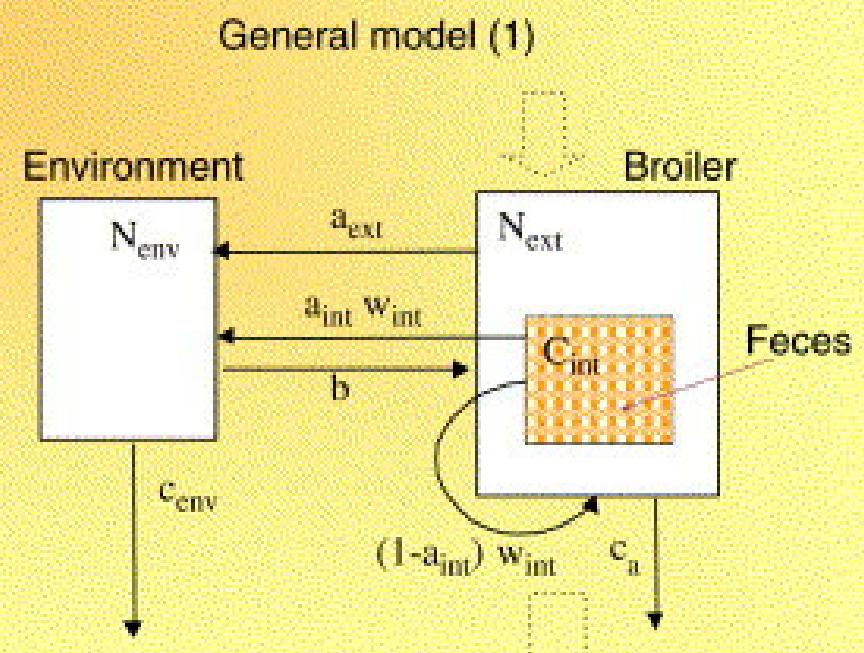

Line of carcasses

Fig. 2. A typical phase in the chicken processing model.

$N_{\text {env }}$ represents the number of campylobacter in the physical environment of the chicken in a processing phase (expressed in cfu, colony forming units). $N_{\text {ext }}$ is the number on the exterior of the chicken, and $C_{\text {int }}$ is the concentration in the intestines, containing the feces. The transfer coefficients are explained in Table 1 and depend on the processing phase $S$.

Table 1.

Transfer coefficients for processing phase $S$

\begin{tabular}{|c|c|}
\hline$a_{\text {ext }, s}$ & $\begin{array}{l}\text { probability per cfu campylobacter on the exterior (skin and feathers) to move from the } \\
\text { carcass exterior to the environment, per processing stage } S \text {. }\end{array}$ \\
\hline$b_{\mathrm{env}, s}$ & $\begin{array}{l}\text { probability per cfu campylobacter in the environment to move from the environment to the } \\
\text { carcass exterior, per processing stage } S \text {. }\end{array}$ \\
\hline$a_{\text {int }, s}$ & $\begin{array}{l}\text { probability per cfu campylobacter in the leaking feces to move to the environment, per } \\
\text { processing stage } S \text {. (With corresponding probability } 1-a_{\text {int, },} \text { per cfu to move from the } \\
\text { interior to the exterior of the carcass directly.) }\end{array}$ \\
\hline$C_{\text {env }, S}$ & $\begin{array}{l}\text { probability of inactivation or removal per cfu campylobacter in the environment which is } \\
\text { not transferred to the carcass exterior, per processing stage } S \text {. }\end{array}$ \\
\hline$W_{\text {int }, s}(i)$ & amount of feces (gram) that leaks from carcass $i$ at processing stage $S$. \\
\hline$C_{\text {ext }, S}$ & $\begin{array}{l}\text { probability of inactivation or removal per cfu campylobacter on the carcass exterior which } \\
\text { is not transferred to the environment, per processing stage } S \text {. }\end{array}$ \\
\hline
\end{tabular}

Mass balance equations are formulated which say, e.g., that the cfu's at the end of phase $S$ on the exterior equals the number at the beginning, minus what is 
Reliability Engineering \& System Safety Volume 91, Issues 10-11, OctoberNovember 2006, Pages 1364-1372 The Fourth International Conference on Sensitivity Analysis of Model Output (SAMO 2004) - SAMO 2004

transferred to the environment or inactivated/removed entirely, plus what moves onto the exterior during phase $S$. For more detail, see [2].

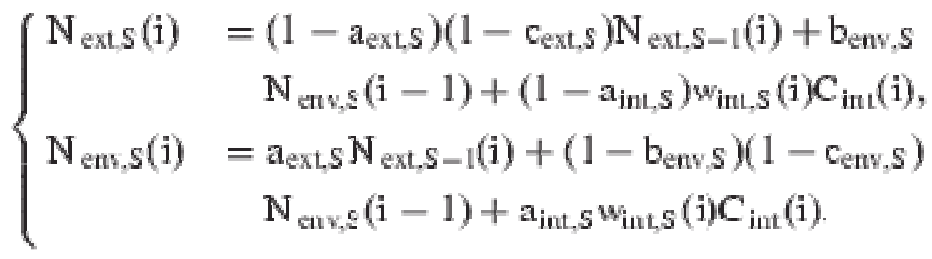

In equilibrium we have $N_{\text {env }, s}(i)=N_{\text {env, } s}(i-1)$, so that

$$
\mathrm{N}_{\text {env }, S}=\frac{\mathrm{a}_{\mathrm{ext}} \mathrm{N}_{\mathrm{ext}, S-1}+\mathrm{a}_{\text {int }} \mathrm{w}_{\text {int }} \mathrm{C}_{\text {int }}}{\mathrm{b}_{\text {env }}+\mathrm{c}_{\mathrm{env}}-\mathrm{b}_{\text {env }} \mathrm{c}_{\mathrm{env}}} .
$$

Ideally, we would like to have numerical values for the coefficients in Table 1. However, experimental data yielding these values are not available. Failing that, we would like to quantify the uncertainty in the transfer coefficients. Expert judgment could be applied for this purpose, if the experts had detailed knowledge of the interactions in each processing phase. Unfortunately, that is not the case.

Experts are however able to quantify their uncertainty regarding the number of cfu's on a broiler in the situations described below taken from the elicitation protocol:

At the beginning of a new slaughtering day a thinned-flock is slaughtered in a "typical large broiler chicken slaughterhouse". Suppose each chicken of this flock to be infected with campylobacter, both externally and internally. We suppose every chicken to be externally infected with $10^{5}$ campylobacters per carcass and internally with $10^{8}$ campylobacters per gram of caecal content at the beginning of each slaughtering stage (a hypothetical situation). So at the beginning of scalding, plucking etc., each chicken has this (identical) external and internal contamination rate.

Question A1. All chickens of the particular flock are passing successively each slaughtering stage. How many campylobacters (per carcass) will be found after each of the mentioned stages of the slaughtering process, each time on the first chicken of the flock?

Experts respond to these questions, for each phase, by stating the $5 \%, 50 \%$ and $95 \%$ quantiles of their uncertainty distributions. A sample from the elicitation protocol is given in the appendix. If distributions on the transfer coefficients in Table 1 are given, then a distributions, per processing phase, for the elicited variables can be computed from the mass balance equations by a Monte Carlo simulation of the following equations (the processing phase $S$ is suppressed in the notation) 
Reliability Engineering \& System Safety Volume 91, Issues 10-11, OctoberNovember 2006, Pages 1364-1372 The Fourth International Conference on Sensitivity Analysis of Model Output (SAMO 2004) - SAMO 2004

$$
\begin{aligned}
& \mathrm{A} 1=10^{3} \times\left(1-\mathrm{a}_{\mathrm{axt}}\right) \times\left(1-\mathrm{c}_{\mathrm{a}}\right) \\
& +10^{8} \times\left(1-a_{\text {int }}\right) \times w_{\text {int }} \times\left(1-r_{a}\right) \text {. } \\
& \mathrm{A} 2=\mathrm{A} 1+\mathrm{b} \times\left(\mathrm{a}_{\mathrm{ext}} \times 10^{5}+\left(\mathrm{a}_{\mathrm{int}}\right)\right. \\
& \left.x \text { wint } \times 10^{8}\right) /\left(b+c_{\text {ent }}-b \times c_{\text {env }}\right) \text {, } \\
& \mathrm{B} 1=10^{4} \times\left(1-\mathrm{a}_{\mathrm{exr}}-\mathrm{c}_{\mathrm{a}}+\mathrm{a}_{\mathrm{exr}} \times \mathrm{c}_{\mathrm{a}}\right) \text {, } \\
& \mathrm{B} 2=\mathrm{B} 1+\mathrm{b} \times \mathrm{a}_{\mathrm{exr}} \times 10^{4} /\left(\mathrm{b}+\mathrm{c}_{\mathrm{e}}-\mathrm{b}^{\mathrm{s}} \mathrm{c}_{\mathrm{e}}\right) \text {, } \\
& \mathrm{C}=\left(1-\mathrm{b}-\mathrm{c}_{\mathrm{e}}+\mathrm{b} \times \mathrm{c}_{\mathrm{e}}\right)^{99} \mathrm{~b} \\
& \times \frac{\left(a_{e x r} 10^{5}+\left(a_{i n t}\right) \times w_{n t} \times 10^{8}\right)}{\left(b+c_{e n v}-b \times c_{e n v}\right)} \\
& \mathrm{W}_{\text {int }}=\mathrm{W}_{\text {int }} \text {. }
\end{aligned}
$$

The variables $A 1, A 2, \ldots, w_{\text {int }}$ are the variables assessed by the experts. Question $A 2$ is given in the appendix. Questions $B 1$ and $B 2$ are similar to $A 1$ and $A 2$, but refer to a flock in which the birds are externally contaminated, but not colonized (internally). Question C asks for the infection on the 100th broiler of an uninfected flock which is processed after an internally and externally infected flock. $W_{\text {int }}$ was queried directly. It is included here to indicate that its distribution must conform to the decision maker's quantile assessments. For the first 3 processing phases, we have 6 equations; for later phases the intestines are removed and the variable $W_{\text {int }}$ is not defined. In total we have 39 such equations, counting the alternative processes for scalding and chilling. ${ }^{1}$ The number of equations is equal to the number of transfer coefficients for the whole line.

Assuming distributions for coefficients on the right-hand sides are known, we could sample from these distributions and build up distributions for the quantities on the left hand side. These quantities are assessed by the experts. We would like these distributions to comply with the quantiles given by the decision maker.

The probabilistic inversion problem may now be expressed as follows: find a joint distribution over the transfer coefficients, such that the quantiles of the quantities on left hand sides of the above equations agree with the decision maker's quantiles. If more than one such joint distribution exists, pick the least informative of these. If no such joint distribution exists, pick a "best fitting" distribution.

\section{Probabilistic inversion}

Let $X$ and $Y$ be $n$ and $m$-dimensional random vectors, respectively, and let $G$ be a function from $\mathscr{h}^{\mathrm{n}}$ to $\mathscr{h}^{\mathrm{m}}$. We call $\mathrm{x} \in \mathscr{R}^{\mathrm{n}}$ an inverse of $y \in \mathscr{h}^{\mathrm{m}}$ under $G$ if $G(x)=y$. Similarly we call $X$ a probabilistic inverse of $Y$ under $G$ if $G(X) \approx Y$, where " $\approx$ " means "has the same distribution as". If $\{\mathbf{Y} \mid \mathbf{Y} \in \mathbf{C}\}$ is the set of random vectors satisfying constraints $C$, then we say that $X$ is an element of the probabilistic inverse of [Y $\mid \mathrm{Y} \in C]$ under $G$ if $G(X) \in C$. Equivalently, and more conveniently, if the distribution of $Y$ is partially specified, then we say that $X$ is a probabilistic inverse of $Y$ under $G$ if $G(X)$ satisfies the partial specification of $Y$. In the current context, the transfer coefficients in Table 1 play the role of $X$, and the left hand sides of Eq. (1) play the role of $Y$. That is 
Reliability Engineering \& System Safety Volume 91, Issues 10-11, OctoberNovember 2006, Pages 1364-1372 The Fourth International Conference on Sensitivity Analysis of Model Output (SAMO 2004) - SAMO 2004

$\mathbf{Y}=\left(\mathrm{A}_{1,1}, \mathrm{~A}_{2,1}, \mathbf{B}_{1}, \mathbf{B}_{2}, \mathrm{C}_{1}, \mathrm{~W}_{\mathrm{int}, 1}, \mathrm{~A}_{1,2}, \ldots, \mathbf{B}_{2,5}, \ldots, \mathrm{C} 5\right)$;

( 39 components in total).

The joint distribution of these variables is partially specified by the decision maker, namely by given $5 \%, 50 \%$ and $95 \%$ quantiles. The right-hand sides of (1) constitute the function $G$.

If the function $G$ could be inverted analytically, then it would be a simple matter to compute $X$ as $G^{-1}(Y)$. Of course this is generally not possible, and we must devise other ways to find $X$. A number of approaches could be considered. A thorough discussion of this problem is found in [8], and a shorter discussion in [7]. By far most satisfactory to date are techniques based on sample reweighting, and these have been applied to the chicken line model.

The strategy for sample re-weighting can be described informally as follows. We first choose an initial distribution for $X$, that is, for the transfer coefficients. This distribution must be chosen such that, when we sample it a large number of times and compute $Y$ via (1), some samples fall within each interquantile interval for each variable in $Y$, and all samples are physically possible. The choice of initial distribution is not further constrained, but it should cover the range of realistic values. We take $N$ samples from $X$ and compute $N$ samples for $Y$, yielding $N$ samples for $(X, Y)$. When drawn from the initial distribution, each of the $N$ samples has probability $1 / N$. We now wish to re-weight these $N$ samples such that, if we re-sample this distribution, drawing each sample (with replacement) with probability given by its weight, then the quantile constraints on $Y$ are satisfied in the re-sampled distribution.

There are various strategies for finding the weights [8]; we give an informal description of two strategies, namely iterative proportional fitting (IPF) and PARameter fitting for uncertain models (PARFUM). These involve iteratively readjusting an initial set of weights so as to satisfy the constraints. For convenience, we describe this for one processing phase with 6 elicitation variables ( $Y$ is restricted to 6 components). Each elicitation variable has 4 interquantile intervals, and the weighted sum of samples falling in each such interval must satisfy the corresponding quantile constraints. Thus, the sum of the weights of all samples in which $A_{1}$ falls beneath the decision maker's $5 \%$ quantile for variable $A_{1}$ should be $5 \%$, etc.

Since each sample contains a value for $\left(A_{1}, A_{2}, B_{1}, B_{2}, C, W_{\text {int }}\right)$, and each component falls in one of 4 interquantile intervals, we may represent this sample as a 6 -vector of components, each component taking values in $\{1,2,3,4\}$. There are $4^{6}=4096$ possible vectors of this type, and we may think of each such vector as an interquantile cell containing a number of samples. Not all cells will be physically possible. It is easy to see from (1) that $A_{1} \leqslant A_{2}$, and $B_{1} \leqslant B_{2}$. Thus, if the $50 \%$ quantile for $A_{1}$ is above the $5 \%$ quantile for $A_{2}$, then it is impossible that $A_{1}$ could be above its median while $A_{2}$ is below its $5 \%$ quantile. Fortunately it is not necessary to figure out which combinations of interquantile intervals are feasible; sampling $X$ and computing $Y$ via (1) does that automatically. It is well to realize, however, that a large number of mathematically possible interquantile cells may actually be unfeasible under the function $G$. In a typical example for a processing phase, we would draw 65,000 samples and find that 150-300 of the 4096 interquantile cells were occupied. The weight assigned to each interquantile cell is simply the total weight of the samples falling in that cell. In our iterative algorithms, two samples falling in the same cell will be treated in the same way; 
Reliability Engineering \& System Safety Volume 91, Issues 10-11, OctoberNovember 2006, Pages 1364-1372 The Fourth International Conference on Sensitivity Analysis of Model Output (SAMO 2004) - SAMO 2004

therefore we can restrict our problem to that of finding weights for the non-empty interquantile cells. When these weights are found, we just distribute the cell weight uniformly over the samples in the cell to get the sample weights.

Rather than describe the IPF and PARFUM algorithms formally, it is appropriate here simply to illustrate them on a simple example and report the relevant mathematical facts. Details can be retrieved from the cited literature. For purposes of illustration, we consider only 2 elicitation variables, each with 4 interquantile intervals corresponding to the $5 \%, 50 \%$ and $95 \%$ quantiles. The interquantile cells can be represented as a $4 \times 4$ matrix; where, for example, a sample is said to fall in cell $(3,2)$ if it is between the $50 \%$ and $95 \%$ quantiles for variable 1 and between the $5 \%$ and $50 \%$ quantiles for variable 2 .

We start with an initial distribution over $X$ and generate an initial distribution over the interquantile cells, which we represent in Table 2 . Note that 6 cells are empty. The marginals are shown in boldface.

Table 2.

Initial distribution over interquantile cells

\begin{tabular}{|l|l|l|l|l|}
\hline 0.1966 & 0.0006 & 0 & 0 & $\mathbf{0 . 1 9 7 2}$ \\
\hline 0.0407 & 0.1642 & 0.005 & 0 & $\mathbf{0 . 2 0 9 9}$ \\
\hline 0 & 0.0094 & 0.1196 & 0.0155 & $\mathbf{0 . 1 4 4 5}$ \\
\hline 0 & 0 & 0.0008 & 0.4476 & $\mathbf{0 . 4 4 8 4}$ \\
\hline $\mathbf{0 . 2 3 7 3}$ & $\mathbf{0 . 1 7 4 2}$ & $\mathbf{0 . 1 2 5 4}$ & $\mathbf{0 . 4 6 3 1}$ & \\
\hline
\end{tabular}

The problem is now to adjust the non-empty cells in Table 2 such that the margins equal $0.05,0.45,0.45,0.05$; which are the probabilities associated with the decision maker's interquantile intervals.

The IPF algorithm was introduced by [9] and rediscovered by [10]. Its convergence properties were studied by many, including [11] and [12]. Simply stated, we first multiply each row by constant, so that the column sums agree with the target. For the first row this constant is target/row sum $=0.05 /(0.1966+0.0006)$. Then we multiply each column by the constant (target/column sum) to make the column sums agree with the target, then again the rows are multiplied by a constant, etc. Fig. 3 illustrates the procedure. The target margins are shown by the starting distribution. This is first adapted to fit the row sums, leaving the column sums unfit. Then the first adaptation is adapted to fit the column sums, thereby unfitting the row sums. Then the row sums are fit again, then the columns, etc. 
Reliability Engineering \& System Safety Volume 91, Issues 10-11, OctoberNovember 2006, Pages 1364-1372 The Fourth International Conference on Sensitivity Analysis of Model Output (SAMO 2004) - SAMO 2004

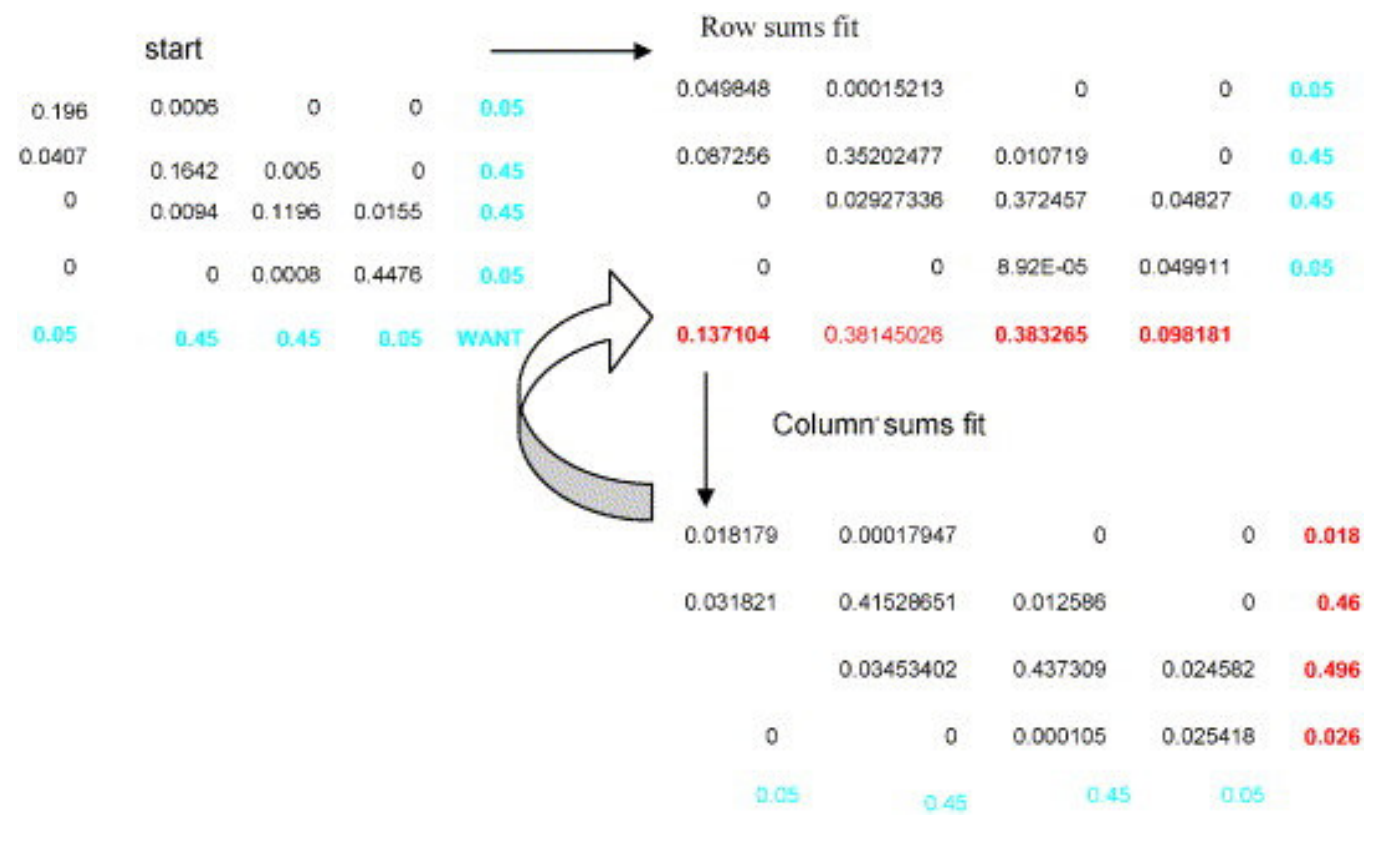

Fig. 3. Iterative proportional fitting.

Csiszar [12] showed that this algorithm converges if there is a distribution whose non-zero cells are a subset of the non-zero cells of the initial distribution which satisfies the target margins. In that case IPF converges to the distribution which has minimum information relative to the starting distribution, in the set of distributions with the target margins. This distribution may have zero's in cells where the starting distribution is non-zero. The result holds for arbitrary finite dimensions, and arbitrary finite numbers of cells per dimension. The target marginal distributions need not be the same on each dimension.

It is evident that the criterion for convergence becomes more difficult to satisfy as the number of zero's increases. When the criterion is not met the probabilistic inversion problem is infeasible and IPF need not converge. In otherwords, there is no distribution over the non-zero cells in the starting matrix which has the target margins. In the case of two dimensions, it is known that IPF oscillates between two distributions, in case of non convergence [13]. Nothing is known about the behavior of IPF in higher dimensions when the condition for convergence is not satisfied.

The PARFUM algorithm [8] and [14] differs from IPF in the following way. Instead of first fitting the row sums, then the column sums, then again the row sums, etc.; PARFUM successively averages the row and column sum fits. It is schematized in Fig. 4: 
Reliability Engineering \& System Safety Volume 91, Issues 10-11, OctoberNovember 2006, Pages 1364-1372 The Fourth International Conference on Sensitivity Analysis of Model Output (SAMO 2004) - SAMO 2004

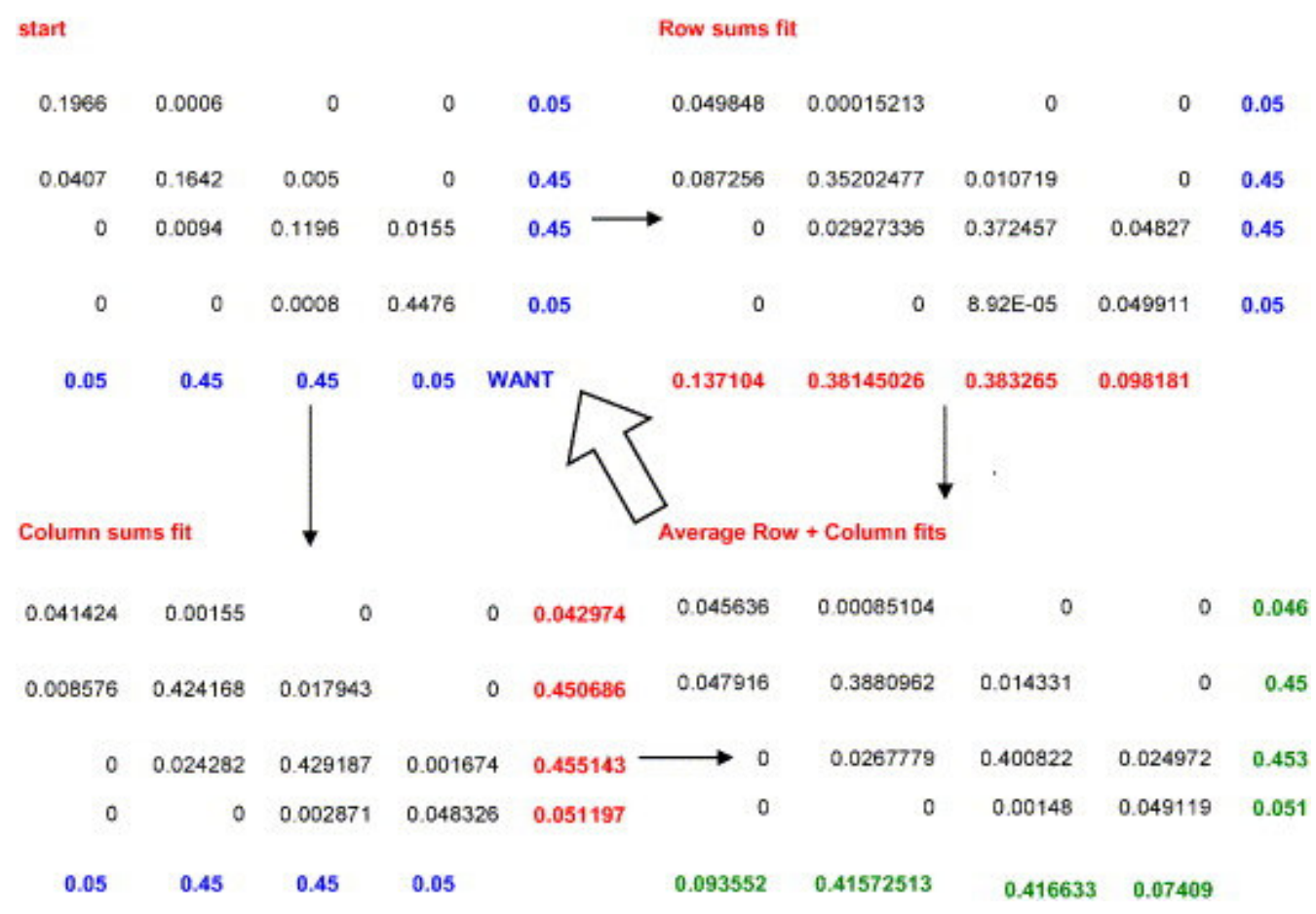

Fig. 4. The PARFUM algorithm.

The stationary points of the PARFUM algorithm minimize the following functional

$\mathbf{F}(\mathbf{P})=\mathbf{I}\left(\mathbf{P}^{\text {row fit }} \mid \mathbf{P}\right)+\mathbf{I}\left(\mathbf{P}^{\text {column fit }} \mid \mathbf{P}\right)$,

relative to the starting distribution. Here, $\mathbf{I}(\mathrm{Q} \mid \mathrm{P})$ denotes the relative information of $Q$ with respect to $P$. The function $F$ decreases on each PARFUM iteration and converges to a minimum. If $P$ is a solution, that is, if $P$ 's row and column fits agree with the target, then $\mathrm{P}^{\text {row fit }}=\mathrm{P}=\mathrm{P}^{\text {column fit }}$, so that $F(P)=0$. Most importantly, if the problem is feasible, then PARFUM converges to a $P$ with $F(P)=0$, that is, it converges to a solution [8]. If the problem is not feasible, PARFUM's stationary points realize the minimum value of $F$. In practice PARFUM is observed to converge in this case also, and an example in which PARFUM actually oscillates between distinct stationary points realizing the same value of $F$ has not yet been found.

In practical experience, IPF is faster than PARFUM, but both are quite fast on modern platforms and easy to implement. If the problem is feasible then IPF is generally preferred. If infeasible, then IPF tends to distribute the lack of fit quite unevenly and tends to concentrate weight on a small number of samples. In such cases PARFUM often gives better results. Of course, if IPF does not cycle, we have no way of knowing on a finite number of iterations whether it is converging. Appeal to common sense is appropriate.

These algorithms have several advantages relative to other methods. First, they are 'dumb' in the sense that they do not require intelligent steering. Second, they avoid computationally expensive matrix manipulations, but simply loop repeatedly through the interquantile cells. Finally, since rows and columns are altered one at a time, the whole sample need not be stored in memory, and there is effectively no limit on the size of problems which can be tackled. There are disadvantages as well. Most significantly, it is impossible in practice to know if IPF is converging 
Reliability Engineering \& System Safety Volume 91, Issues 10-11, OctoberNovember 2006, Pages 1364-1372 The Fourth International Conference on Sensitivity Analysis of Model Output (SAMO 2004) - SAMO 2004

without verifying the condition for convergence, and this is just as hard as finding the solution. PARFUM has a distinct advantage in this regard. In case of infeasibility neither algorithm yields information on how the original sample might be extended to yield better solutions.

\section{Results}

The results with the model described above yielded a very poor fit between the re-predicted and decision maker distributions for some variables. Table 3 shows the results for defeathering. Especially bad fits are circled.

Inspection of the experts rationales revealed that the experts distinguished transfer mechanisms from the exterior to the environment. Campylobactor in the pores of the skin would be difficult to remove, but on the feathers or skin surface they would come off more easily. It therefore makes a difference whether the birds have been contaminated during transport only (giving rise to only contamination of the exterior) or at the farm (resulting in intestinal colonization and contamination on the exterior. These two different situations had been the starting point of questions $A$ and $B$. The processing model was therefore altered to include this second transport pathway, as shown in Fig. 5 . The coefficient $a_{\text {ext }}$ is replaced by two coefficients, $a_{x a}$ and $a_{x b}$. The equations for the elicited quantities now become:

$$
\begin{aligned}
& A 1=10^{5} \times\left(1-a_{\text {xa }}\right) \times\left(1-c_{\mathrm{a}}\right)+10^{8} \times\left(1-a_{\text {int }}\right) \times w_{\text {int }} \times(1- \\
& \left.c_{\mathrm{a}}\right), A 2=A 1+b \times\left(a_{\mathrm{xa}} \times 10^{5}+\left(a_{\text {int }}\right) \times w_{\text {int }} \times 10^{8} /\left(b+c_{\text {env }}-b \times c_{\text {env }}\right), B 1=10^{4} \times\left(1-a_{\mathrm{xb}}-\right.\right. \\
& \left.c_{\mathrm{a}}+a_{\mathrm{xb}} \times c_{\mathrm{a}}\right), B 2=B 1+b \times a_{\mathrm{xb}} \times 10^{4} /\left(b+c_{\mathrm{e}}-b \times c_{\mathrm{e}}\right), C=(1-b- \\
& \left.c_{\mathrm{e}}+b \times c_{\mathrm{e}}\right)^{99} \times b \times\left(a_{\mathrm{xa}} \times 10^{5}+\left(a_{\text {int }}\right) \times w_{\text {int }} \times 10^{8}\right) /\left(b+c_{\text {env }}-b \times c_{\text {env }}\right), W_{\text {int }}=W_{\text {int }} .
\end{aligned}
$$

[Note that parameter symbols are not identical throughout the text, like $c_{a}=c_{\text {ext }}$, etc.] 
Reliability Engineering \& System Safety Volume 91, Issues 10-11, OctoberNovember 2006, Pages 1364-1372 The Fourth International Conference on Sensitivity Analysis of Model Output (SAMO 2004) - SAMO 2004

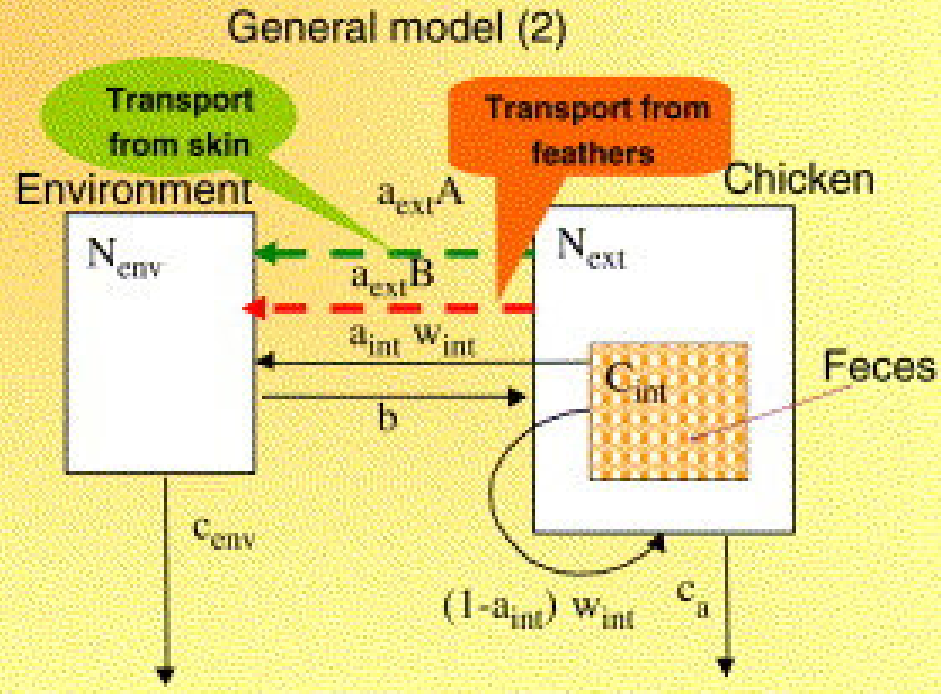

Fig. 5. Processing model with additional transport pathway [from skin is tightly attached $(/)$, from feathers = loosely attached].

With the second model, the probabilistic inversion yielded better fits, as shown in Table 4 (this is due to better agreement with the experts' mental model, and to the fact that there is now an extra transfer coefficient).

Table 4.

Re-predictions with model (2)

\begin{tabular}{|c|l|l|l|l|l|l|l|}
\hline Variables & Quantile & \multicolumn{2}{|l|}{ Scalding low } & \multicolumn{2}{l|}{ Scalding intermediate } & \multicolumn{2}{l|}{ Defeathering } \\
\hline & & IPF & PARFUM & IPF & PARFUM & IPF & PARFUM \\
\hline (a) $A 1$ & 0.05 & 0.05 & 0.05 & 0.04 & 0.04 & 0.02 & 0.05 \\
\hline & 0.5 & 0.50 & 0.53 & 0.42 & 0.45 & 0.78 & 0.49 \\
\hline & 0.95 & 0.95 & 0.95 & 0.81 & 0.86 & 0.97 & 0.94 \\
\hline$A 2$ & 0.05 & 0.05 & 0.04 & 0.07 & 0.07 & 0.00 & 0.04 \\
\hline & 0.5 & 0.50 & 0.41 & 0.70 & 0.65 & 0.14 & 0.38 \\
\hline & 0.95 & 0.95 & 0.93 & 0.95 & 0.97 & 0.91 & 0.72 \\
\hline & 0.05 & 0.05 & 0.05 & 0.05 & 0.05 & 0.02 & 0.04 \\
\hline & 0.5 & 0.50 & 0.50 & 0.50 & 0.50 & 0.20 & 0.36 \\
\hline & 0.95 & 0.95 & 0.95 & 0.95 & 0.95 & 0.39 & 0.70 \\
\hline
\end{tabular}


Reliability Engineering \& System Safety Volume 91, Issues 10-11, OctoberNovember 2006, Pages 1364-1372 The Fourth International Conference on Sensitivity Analysis of Model Output (SAMO 2004) - SAMO 2004

\begin{tabular}{|c|c|c|c|c|c|c|c|c|c|}
\hline \multirow[t]{2}{*}{ Variables } & \multirow[t]{2}{*}{ Quantile } & \multicolumn{2}{|c|}{ Scalding low } & \multicolumn{2}{|c|}{ Scalding intermediate } & \multicolumn{2}{|c|}{ Defeathering } & & \\
\hline & & IPF & PARFUM & IPF & PARFUM & IPF & PARFUM & & \\
\hline \multirow[t]{3}{*}{$B 2$} & 0.05 & 0.05 & 0.05 & 0.05 & 0.05 & 0.25 & 0.29 & & \\
\hline & 0.5 & 0.50 & 0.50 & 0.50 & 0.50 & 0.43 & 0.64 & & \\
\hline & 0.95 & 0.95 & 0.95 & 0.95 & 0.95 & 0.93 & 0.96 & & \\
\hline \multirow[t]{3}{*}{$C 1$} & 0.05 & 0.05 & 0.08 & 0.05 & 0.05 & 0.01 & 0.08 & & \\
\hline & 0.5 & 0.50 & 0.54 & 0.58 & 0.46 & 0.44 & 0.51 & & \\
\hline & 0.95 & 0.95 & 0.96 & 0.94 & 0.88 & 0.99 & 0.94 & & \\
\hline \multirow[t]{3}{*}{$W_{\text {int }}$} & 0.05 & 0.05 & 0.06 & 0.05 & 0.05 & 0.05 & 0.13 & & \\
\hline & 0.5 & 0.50 & 0.54 & 0.50 & 0.48 & 0.50 & 0.55 & & \\
\hline & 0.95 & 0.95 & 0.96 & 0.95 & 0.91 & 0.95 & 0.96 & & \\
\hline \multirow[t]{2}{*}{ Variables } & Quantile & \multicolumn{2}{|c|}{ Evisceration } & \multicolumn{2}{|c|}{ Washing } & \multicolumn{2}{|c|}{ Cooling air } & \multicolumn{2}{|c|}{ Cooling spray } \\
\hline & & IPF & PARFUM & IPF & PARFUM & IPF & PARFUM & IPF & PARFUM \\
\hline \multirow[t]{3}{*}{ (b) $A 1$} & 0.05 & 0.05 & 0.05 & 0.05 & 0.05 & 0.00 & 0.04 & 0.05 & 0.05 \\
\hline & 0.5 & 0.50 & 0.51 & 0.50 & 0.51 & 0.05 & 0.39 & 0.53 & 0.50 \\
\hline & 0.95 & 0.95 & 0.95 & 0.95 & 0.95 & 0.95 & 0.96 & 1.00 & 0.98 \\
\hline \multirow[t]{3}{*}{$A 2$} & 0.05 & 0.05 & 0.05 & 0.05 & 0.05 & 0.05 & 0.39 & 0.05 & 0.06 \\
\hline & 0.5 & 0.50 & 0.49 & 0.50 & 0.49 & 0.50 & 0.67 & 0.50 & 0.51 \\
\hline & 0.95 & 0.95 & 0.95 & 0.95 & 0.95 & 0.95 & 0.96 & 0.95 & 0.94 \\
\hline \multirow[t]{3}{*}{$B 1$} & 0.05 & 0.05 & 0.05 & 0.05 & 0.05 & 0.01 & 0.04 & 0.05 & 0.06 \\
\hline & 0.5 & 0.50 & 0.50 & 0.51 & 0.51 & 0.05 & 0.39 & 0.53 & 0.54 \\
\hline & 0.95 & 0.95 & 0.95 & 0.95 & 0.95 & 0.91 & 0.94 & 1.00 & 0.98 \\
\hline \multirow[t]{3}{*}{$B 2$} & 0.05 & 0.05 & 0.05 & 0.05 & 0.05 & 0.00 & 0.04 & 0.05 & 0.04 \\
\hline & 0.5 & 0.50 & 0.50 & 0.50 & 0.49 & 0.05 & 0.39 & 0.50 & 0.44 \\
\hline & 0.95 & 0.95 & 0.95 & 0.95 & 0.95 & 0.90 & 0.93 & 0.95 & 0.92 \\
\hline \multirow[t]{3}{*}{$C 1$} & 0.05 & 0.05 & 0.05 & $\%$ & 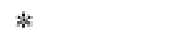 & 0.05 & 0.05 & 0.05 & 0.05 \\
\hline & 0.5 & 0.50 & 0.50 & * & $*$ & 0.50 & 0.50 & 0.50 & 0.49 \\
\hline & 0.95 & 0.95 & 0.95 & * & $*$ & 0.95 & 0.95 & 0.95 & 0.93 \\
\hline \multirow[t]{3}{*}{$W_{\text {int }}$} & 0.05 & 0.05 & 0.05 & 수소 & 수수 & 44 & 수수 & -4 & 수 수 \\
\hline & 0.5 & 0.50 & 0.50 & 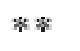 & H⿻ & $\omega *$ & Wh & $=\%$ & 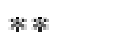 \\
\hline & 0.95 & 0.95 & 0.95 & 4 & 45 & 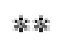 & 4 & $\Rightarrow$ & $s$ \\
\hline
\end{tabular}

* Degenerate distribution.

$*$ Not present in this phase. 
Reliability Engineering \& System Safety Volume 91, Issues 10-11, OctoberNovember 2006, Pages 1364-1372 The Fourth International Conference on Sensitivity Analysis of Model Output (SAMO 2004) - SAMO 2004

The bold values indicate the solution chosen. We see that the PARFUM solution was chosen in 3 of the seven cases. There is still lack of fit, in particular for defeathering and cooling air. However, overall, the model revision has produced a better fit. It is a truism that no model is fully adequate to reality. Information regarding the degree and locus of misfit is extremely valuable. The methods discussed here provide such information. The alternative is to search for compliant experts who will assess model parameters directly (and often anonymously); this cannot lead to model improvement.

\section{Conclusions}

In risk modelling, we must frequently employ expert judgment to assess model parameters which are not directly observable, and about which experimental evidence is lacking. The model under consideration may be new and even unknown to the experts. In such situations it is impossible to query experts directly about parameters in the model. We can ask experts about quantities predicted by the model with which they have some experience and some feeling. The problem then arises, how we should pull the experts' uncertainty distributions on the query variables back onto the parameters of the model. This problem is termed probabilistic inversion. Iterative sample re-weighting methods are available to solve such problems, as illustrated in the model of chicken processing lines. IPF and PARFUM are easy to implement and have a theoretical foundation. They provide useful tools for the practicing risk modeller.

The present study illustrates a fruitful interaction between the modellers and the experts. The first model discussed above lacked an important transfer mechanism, and this caused a serious lack of fit between the experts' distributions and the re-predicted distributions emerging after probabilistic inversion. Studying the experts' rationales led to a revision of the model and a good fit between the experts' and the re-predicted distributions.

\section{References}

[1] H. Rosenquist, N.L. Nielsen, H.M. Sommer, B. Norrung and B.B. Christensen, Quantitative risk assessment of human campylobacteriosis associated with thermophilic campylobacter species in chickens, Int J Food Microbiol 83 (2003) (1), pp. 87-103.

[2] M. Nauta, I. Van der Fels-Klerx and A. Havelaar, A poultry-processing model for quantitative microbiological risk assessment, Risk Anal 25 (2005), pp. 85-98.

[3] H.J. Van der Fels-Klerx, R.M. Cooke, M.N. Nauta, L.H. Goossens and A.H. Havelaar, A structured expert judgment study for a model of campylobacter contamination during broiler-chicken processing, Risk Anal 25 (2005), pp. 109124.

[4] B.C.P. Kraan and R.M. Cooke, Processing expert judgements in accident consequence modelling, Radiat Prot Dosim 90 (2000) (3), pp. 311-315.

[5] B.C.P. Kraan and R.M. Cooke, Uncertainty in compartmental models for hazardous materials-a case study, J Hazar Mater 71 (2000), pp. 253-268. Abstract | PDF (251 K) | Abstract + References in Scopus | Cited By in Scopus 
Reliability Engineering \& System Safety Volume 91, Issues 10-11, OctoberNovember 2006, Pages 1364-1372 The Fourth International Conference on Sensitivity Analysis of Model Output (SAMO 2004) - SAMO 2004

[6] Kraan BCP. Probabilistic inversion in uncertainty analysis and related topics. PhD dissertaition, Department of Mathematics, Delft University of Technology, 2002.

[7] Kurowicka D, Cooke RM. Techniques for generic probabilistic inversion. In: Bonano EJ, Camp AL, Majors MJ, Thompson RA, editors, Probabilistic safety assessment and management. Amsterdam: Elsevier, 2002. p. 1543-50.

[8] Du C, Kurowicka D, Cooke RM. Techniques for generic probabilistic inversion. Delft Institute for Applied Mathematics, Comput Stat Data Anal, 2003, to appear.

[9] J. Kruithof, Telefoonverkeersrekening, De Ingenieur 52 (1937) (8), pp. E15E25.

[10] W.E. Deming and F.F. Stephan, On a last squares adjustment of sampled frequency table when the expected marginal totals are known, Ann Math Statist 11 (1940), pp. 427-444. MathSciNet

[11] S.E. Fienberg, An iterative procedure for estimation in contingency tables, Ann Math Statist 41 (1970), pp. 907-917. MathSciNet

[12] I. Csiszar, I-divergence geometry of probability distributions and minimization problems, Ann Probab 3 (1975), pp. 146-158. MathSciNet

[13] Csiszar I, Tusnady G. Information geometry and alternating minimization procedures. Stat Decis 1983 (Suppl 1):205-37.

[14] R.M. Cooke, Parameter fitting for uncertain models: modelling uncertainty in small models, Reliab Eng Syst Saf 44 (1994), pp. 89-102.

[15] Cooke RM, Hora S, Young M, Pasler-Ssauer J, Miller L, Kraan B, Lui C, McKay M, Helton J, Jones A, Harper F, Goossens L. Joint USNRC \CEC consequence uncertainty study: Summary of objectives, approach, application, and results for the dispersion and deposition uncertainty assessment. Technical report vol. III, NUREG/CR-6244, EUR 15755 EN, SAND94-1453, 1994.

[16] B.C.P. Kraan and R.M. Cooke, Post processing techniques for the joint $\mathrm{cec} /$ usnrc uncertainty analysis of accident consequence codes, Statis Comput Simul 57 (1997), pp. 243-259. Abstract + References in Scopus | Cited By in Scopus - 
Reliability Engineering \& System Safety Volume 91, Issues 10-11, OctoberNovember 2006, Pages 1364-1372 The Fourth International Conference on Sensitivity Analysis of Model Output (SAMO 2004) - SAMO 2004

\section{Appendix. Sample of elicitation protocol}

The assumptions in short:

- "Typical large broiler chicken slaughterhouse" in the Netherlands.

- Average day of the year (all seasons).

- At the beginning of the new slaughtering day campylobacter is absent in the slaughterhouse.

- All machines are functioning well.

- Thinned-flocks of 10,000 chickens each.

- The chickens of the flocks are uniform in size and weight.

- The broiler house might have been clean (dry) or dirty (wet).

- Time of fasting is $6 \mathrm{~h}$, travelling time 3 and $4 \mathrm{~h}$ and waiting time is ca. $2 \mathrm{~h}$.

- In the scalding tank the principle of counter current is used.

- Low scalding temperature is $50-52{ }^{\circ} \mathrm{C}$ and intermediate scalding temperature is $55-56{ }^{\circ} \mathrm{C}$.

Part A-A positive flock: At the beginning of a new slaughtering day a thinnedflock is slaughtered in a "typical large broiler chicken slaughterhouse". Suppose each chicken of this flock to be infected with campylobacter, both externally and internally. We suppose every chicken to be externally infected with $10^{5}$ campylobacters per carcass and internally with $10^{8}$ campylobacters per gram of coecal content at the beginning of each slaughtering stage (a hypothetical situation). So at the beginning of scalding, plucking, etc., each chicken has this (identical) external and internal contamination rate.

Question A1: All chickens of the particular flock are passing successively each slaughtering stage. How many campylobacters (per carcass) will be found after each of the mentioned slaughtering stages, each time on the first chicken of this flock?

Number of campylobacters (per carcass) on the first chicken of the flock after/at the end of each slaughtering stage.

\begin{tabular}{|l|l|l|l|}
\hline Slaughtering stage & 5 th & 50 th & 95 th \\
\hline 1. Scalding & & & \\
\hline Low & & & \\
\hline Intermediate & & & \\
\hline 2. Plucking & & & \\
\hline 3. Evisceration & & & \\
\hline 4. Washing & & & \\
\hline
\end{tabular}


Reliability Engineering \& System Safety Volume 91, Issues 10-11, OctoberNovember 2006, Pages 1364-1372 The Fourth International Conference on Sensitivity Analysis of Model Output (SAMO 2004) - SAMO 2004

\begin{tabular}{|l|l|l|l|}
\hline 5. Chilling & & & \\
\hline Complete air-chilling & & & \\
\hline Spray-chilling & & & \\
\hline
\end{tabular}

Question A2: All chickens of the particular flock are passing successively each slaughtering stage. How many campylobacters (per carcass) are found after each of the mentioned slaughtering stages, each time on the last chicken of this flock?

Number of campylobacters (per carcass) on the final chicken of the flock after/at the end of each slaughtering stage.

\begin{tabular}{|l|l|l|l|}
\hline Slaughtering stage & 5th & 50th & 95 th \\
\hline 1. Scalding & & & \\
\hline Low & & & \\
\hline Intermediate & & & \\
\hline 2. Plucking & & & \\
\hline 3. Evisceration & & & \\
\hline 4. Washing & & & \\
\hline 5. Chilling & & & \\
\hline Complete air-chilling & & & \\
\hline Spray-chilling & & & \\
\hline
\end{tabular}

Part B-An externally infected flock: At the beginning of a new slaughtering day a thinned-flock is slaughtered in a typical large broiler chicken slaughterhouse in the Netherlands. Suppose every chicken of this flock to be externally infected with campylobacter. Internally all chickens are free from campylobacter. Suppose each chicken to have an external infection with $10^{4}$ campylobacters per carcass at the beginning of each slaughtering stage (a hypothetical situation). So, at the beginning of scalding, plucking etc. each chicken has this (identical) external contamination rate.

Question B1: All chickens of the particular flock are passing successively each slaughtering stage. How many campylobacters (per carcass) are found after each of the mentioned slaughtering stages, each time on the first chicken of this flock?

Number of campylobacters (per carcass) on the first chicken of the flock after/at the end of each slaughtering stage.

\begin{tabular}{|l|l|l|l|}
\hline Slaughtering stage & 5 th & 50th & 95 th \\
\hline 1. Scalding & & & \\
\hline Low & & & \\
\hline
\end{tabular}


Reliability Engineering \& System Safety Volume 91, Issues 10-11, OctoberNovember 2006, Pages 1364-1372 The Fourth International Conference on Sensitivity Analysis of Model Output (SAMO 2004) - SAMO 2004

\begin{tabular}{|l|l|l|l|}
\hline Intermediate & & & \\
\hline 2. Plucking & & & \\
\hline 3. Evisceration & & & \\
\hline 4. Washing & & & \\
\hline 5. Chilling & & & \\
\hline Complete air-chilling & & & \\
\hline Spray-chilling & & & \\
\hline
\end{tabular}

Question B2: All chickens of the particular flock are passing successively each slaughtering stage. How many campylobacters (per carcass) are found after each of the mentioned slaughtering stages, each time on the last chicken of this flock?

\begin{tabular}{|l|l|l|l|}
\hline $\begin{array}{l}\text { Number of campylobacters (per carcass) on the last chicken of the flock after/at the end of each } \\
\text { slaughtering stage. }\end{array}$ & 5th & 50 th & 95th \\
\hline Slaughtering stage & & & \\
\hline 1. Scalding & & & \\
\hline Low & & & \\
\hline Intermediate & & & \\
\hline 2. Plucking & & & \\
\hline 3. Evisceration & & & \\
\hline 4. Washing & & & \\
\hline 5. Chilling & & & \\
\hline Complete air-chilling & & & \\
\hline Spray-chilling & & & \\
\hline
\end{tabular}

${ }^{1}$ The decision maker considered variable $C$ for washing degenerate, i.e. zero with probability 1 . Removing this would give 38 equations. 